\title{
Literaturas nacionales, literaturas supranacionales: el lugar de los Estudios Ibéricos ${ }^{1}$
}

\author{
SANTIAGO PÉREZ ISASI
}

\begin{abstract}
National Literatures, Supranational Literatures: the Position of Iberian Studies. In this article I defend the need for the existence of the scientific field of Iberian Studies which deals with Spanish and Portuguese literary history from a supranational and not from a merely comparative (in the most traditional sense of the word) perspective. At the same time, I propose that the foundations for these Iberian Studies are not in any way a recent invention; instead, they respond to the same need to overcome strictly national literary histories, a need that was already present as early as the $19^{\text {th }}$ century.
\end{abstract}

Keywords: Iberian literatures, Iberian studies, literary history, comparative literature, national identity

Los primeros años del siglo XXI han visto el nacimiento, o mejor dicho, la consolidación de una nueva disciplina: los Estudios Ibéricos, que en otro lugar he definido como "the consistent and deliberate consideration of the Iberian Peninsula as an interconnected, multilingual and multicultural political, identitarian and (of course) literary polysystem" (Pérez Isasi y Fernandes 2013: 11). Publicaciones recientes a ambos lados del Atlántico (Epps y Fernández Cifuentes 2005; Resina 2009; Martín-Estudillo y Spadaccini 2010; Buffery, Davis y Hooper 2007; Magalhães 2007; Pérez Isasi y Fernández 2013, entre muchas otras) a las que pronto se sumarán varias más actualmente en preparación, indican que se trata de un campo en crecimiento y con una evidente vitalidad académica. Existen, no obstante, varios aspectos aún precarios en cuanto a la configuración epistemológica del campo y su lugar en los organigramas académicos o científicos: así, por ejemplo, los Estudios Ibéricos pueden ser considerados como una sección específica de Literatura Comparada, o bien de los

1 El presente trabajo ha sido desarrollado gracias a una beca de investigación postdoctoral de la Fundação para a Ciência e a Tecnologia de Portugal, para la elaboración del proyecto "Portugal na Ibéria: mapa das relações literárias peninsulares (18701930)” (Ref: SFRH/BPD/78570/2011). 
Literaturas nacionales, literaturas supranacionales: el lugar de los Estudios Ibéricos

Cultural Studies o de las renacidas Area Studies (Pinheiro 2013). También son necesarias reflexiones de calado sobre el modo en que esta disciplina se relaciona con otros conceptos y enfoques que actualmente se sitúan en el centro de los debates de la Teoría de la Literatura, como puede ser el de Literatura Mundial o Literatura-Mundo.

Es evidente que, tal y como la concebimos en este trabajo, los Estudios Ibéricos son una manifestación de la perspectiva supranacional de la Literatura Comparada actual, aplicada a los estudios literarios peninsulares, no en su sentido de mera contraposición dual de dos sistemas, sino como la comprensión rizomática de las interrelaciones culturales ibéricas. En este sentido, cabe considerar los Estudios Ibéricos como un ejemplo de lo que Helena Buescu denomina la "visión prismática" de la Literatura Comparada:

A perspectiva eminentemente comparatista que aqui defendo da literaturamundo permite passar de uma visão constrastiva e binária (por exemplo Eça e Flaubert, ou Eça e Machado, o famoso 'e' da primitiva tradição comparatista) a uma visão mais prismática, em que não faz sentido um gesto exclusivista. (Buescu 2013: 16)

También será útil recordar que, tal y como Claudio Guillén proponía, los Estudios Ibéricos, como disciplina supranacional, no son una mera suma o yuxtaposición de las anteriores configuraciones historiográficas nacionales: ${ }^{2}$ suponen, en cambio, la constatación de que existen en las literaturas producidas en el espacio ibérico fenómenos que no responden a esquemas explicativos tradicionales, porque se sitúan en los márgenes o en los intersticios de las literaturas nacionales. El polisistema ${ }^{3}$ social, político, literario y cultural ibérico, tal y como lo hemos definido, está compuesto de hecho por entidades colectivas (singularmente España y Portugal, pero también Cataluña, Galicia o el País Vasco, así como diversas entidades históricas y políticas hoy desaparecidas) en

2 "Prefiero no decir, con otros, sin más contemplaciones, que la Literatura Comparada consiste en el examen de las literaturas desde un punto de vista internacional. Pues su identidad no depende solamente de la actitud o postura del observador. Es fundamental la contribución palpable a la historia, o al concepto de literatura, de unas clases y categorías que no son meramente nacionales. [...] Y digo supranacional, mejor que internacional, para subrayar que el punto de arranque no lo constituyen las literaturas nacionales, ni las interrelaciones que hubo entre ellas." (Guillén 1985: 13-14)

3 Empleo aquí el término propuesto por Itamar Even-Zohar, de gran utilidad en el análisis de relaciones literarias y culturales transnacionales como las que nos ocupan. Vid. Even-Zohar 1999. 
PÉREZ ISASI

ocasiones superpuestas y en ocasiones enfrentadas, pero siempre fuertemente entrelazadas. ${ }^{4}$

Pero aunque esta visión sobre la unidad-en-la-diversidad de las literaturas y las culturas ibéricas haya ganado especial predicamento en el ámbito académico en la última década, eso no significa que carezca de precedentes críticos o historiográficos. Es mi hipótesis de partida, de hecho, que esta conceptualización de la Península Ibérica como espacio cultural conjunto, aunque con peculiaridades nacionales y regionales, estaba ya presente en la historiografía literaria escrita a lo largo del siglo XIX, y en especial a partir del año 1870; pero que la visión más estrictamente nacional de las literaturas ibéricas se impuso definitivamente en el primer tercio del siglo XX y perduró durante el largo periodo de gobiernos autocráticos (Salazarismo y Franquismo), dominando de hecho, casi hasta nuestros días, la conceptualización histórica, académica y científica de las literaturas peninsulares.

Recordemos, en primer lugar, que la historia literaria de la Península Ibérica es, en sus inicios modernos a comienzos del siglo XIX, un producto del Romanticismo europeo, especialmente alemán y anglosajón, íntimamente vinculado con el surgimiento de los nacionalismos europeos (Aullón de Haro 2008: 20). Este origen exógeno condiciona la apreciación de Iberia, no solo como un conjunto con similitudes y continuidades, sino también como un espacio exótico, desconocido, atractivo y "meridional" y "oriental” al mismo tiempo (Domínguez 2006). De hecho, la división de las naciones europeas en "septentrionales y meridionales", que está presente en las muy influyentes obras de los hermanos Schlegel, ${ }^{5}$ y algo modificada en De l'Allemagne de Madame de Stäel, ${ }^{6}$ condiciona el modo en el que se construyen las primeras historias literarias del siglo XIX. Así, por ejemplo, la obra De la litterature du Midi de L'Europe, de Simonde de Sismondi, realiza una historia de las literaturas del Sur de Europa (incluidas la española y la portuguesa), no por una mera cuestión espacial, sino

4 Este especial entrelazamiento de las literaturas y culturas ibéricas no implica, obviamente, una negación de su interrelación, en ocasiones muy estrecha, con otros espacios literarios y culturales, europeos o trasatlánticos especialmente.

5 "La poesía de los países católicos, la española, la italiana y la portuguesa, forman en [la Edad Media] un conjunto íntimamente unido” (Schlegel 1843, II: 85).

6 "On peut rapporter l'origine des principales nations de l'Europe à trois grandes races différentes: la race latine, la race germanique et la race esclavonne. Les Italiens, les Français, les Espagnols, ont reçu des Romains leur civilisation et leur langage; les Allemands, les Suisses, les Anglais, les Suédois, les Danois et les Hollandais sont des peuples teutoniques; enfin, parmi les Esclavons, les Polonais et les Russes occupent le premier rang. Les nations dont la culture intellectuelle est d'origine latine sont plus anciennement civilisées que les autres.” (Stäel 1813: I, 45) 
Literaturas nacionales, literaturas supranacionales: el lugar de los Estudios Ibéricos

por considerar que existe, entre ellas, una unidad fundamental que permite realizar esta unificación:

Je n'ai pu cependant exécuter qu'une partie du plan que je m'étais d'abord proposé. Il s'étendait à toute l'Europe, et je n'ai parlé que des peuples du Midi de cette contrée. Mais ces derniers forment un ensemble que j'ai cru pouvoir détacher des peuples du Nord. (Simonde de Sismondi 1813-4: I, ii-iii)

De hecho, incluso aquellas historias literarias que tratan separadamente de la literatura española y de la portuguesa, como es el caso de la Geschichte der Poesie und Beredsamkeit seit dem Ende des dreizehnten Jahrhunderts, publicada por Friedrich Bouterwek originalmente entre 1801 y 1819, hacen mención a una unidad esencial que relaciona a ambas literaturas ibéricas:

Así, se unieron españoles y portugueses desde los inicios de su cultura en uno y el mismo tipo de forma y espíritu poéticos. No obstante, lo que de diferente y peculiar tiene la bella literatura de ambas naciones lo mostrarán entre otros los libros que siguen. (Bouterwek 1829, I: 24)

Esta tendencia hacia la consideración unitaria, o al menos interrelacionada, de las literaturas producidas en España y Portugal (aunque no únicamente en español y portugués) quedó en cierta medida abandonada o soterrada cuando la labor de construcción de la historiografía literaria comenzó a ser desarrollada por los propios españoles y portugueses. Se acentúa, por lo tanto, en torno a los años 1840-50 la tendencia a la centralización y a la homogeneización cultural en pro de la construcción de un estado nacional, de forma que la literatura prototípicamente española (por ejemplo) no solo se define como "la literatura escrita en España", sino, fundamentalmente, "la literatura escrita en español", y que responda además a un conjunto de requisitos culturales, religiosos o morales (Pérez Isasi 2013). No quiere esto decir que se elimine absolutamente toda literatura que no responda a estos cánones (por ejemplo, la literatura escrita en otras lenguas peninsulares, o la literatura considerada "heterodoxa"), sino que la organización y configuración del canon responde a estos criterios, privilegiando un determinado modelo de literatura nacional que, según el ideario romántico y nacionalista, responde al "espíritu del pueblo" o Volksgeist.

Así sucede incluso en aquellas historias literarias en las que se da mayor cabida a otras voces distintas a la hegemónica. Este es, por ejemplo, el caso de la Historia crítica de la literatura española, de Amador de los Ríos, una obra ambiciosa que pretendía abarcar toda la literatura producida en el territorio español, en cualquier lengua y en cualquier época (incluyendo latín, árabe y hebreo, 
PÉREZ ISASI

además de español, catalán, español...). Pues bien, incluso en una obra con esta amplia visión de la literatura española, se advierte la tendencia hacia la centralización y la homogeneización:

...a pesar de la vitalidad que todavía entrañaba la nacionalidad catalana, y verdes aún los brillantes lauros ganados por sus más celebrados poetas, comienza a recibirse en aquellas regiones, y dentro de su propio parnaso, la influencia de la lengua y del arte, que florecen en la España Central, augurando ya claramente, conforme dejamos advertido, la grande unidad literaria, cuya realización no estaba en verdad muy lejana. (Amador de los Ríos 1861-5: VI, 487)

En el caso portugués, la exclusión nacionalista de elementos "extraños" en el cuerpo del canon adquiere, particularmente, la forma de una defensa contra lo español, ya sea contra las influencias estilísticas del barroco gongorino ${ }^{7}$, contra el dominio político-cultural ejercido por España durante la Monarquía Dual (1580-1640) ${ }^{8}$ o contra los propios autores portugueses que, en especial durante los siglos XVI y XVII, compusieron su obra total o parcialmente en castellano. La expresión más extrema de esta defensa nacionalista del canon consiste en la eliminación completa de estos autores:

Por esta difficuldade de encontrar os livros necesarios é que prescindi n'este Ensaio de mencionar os Poetas, que só escreveram em latin, e em hespanhol, bem que entre estes haja muitos de grande merecimento, e cujas obras possuo, ou tenho visto: mas fallando em rigor, esta falta nao deve tornar-se mui sensivel, porque Poetas, que só escreveram em verso Latino, ou Castelhano, posto que nacessem em Portugal, nao sam Poetas Portuguezes. (Lopes de Mendonça 1849: 5-6)

Se trata, por lo tanto, de un impulso nacionalizador (en un sentido hegemónico y homogeneizante) de las literaturas española y portuguesa, que provoca,

\footnotetext{
"A corrupção do gosto que já assignalamos na introducção a esta epocha, e a fatal influencia que a eschola de Gongora exerceu sobre a poesia portugueza fez-se sentir de modo bem manifesto no genero lyrico e suas diversas especies." (Fernandes Pinheiro 1862: 186)

8 "Tínhamos perdido a independência; perdemos logo o espírito nacional, o timbre, o amor pátrio (que amor da pátria poderá haver em quem pátria já não tem); a lisonja servil, a adulação infame levou nossos desonrados avós a desprezar seu próprio riquíssimo e tão suave idioma, para escrever no gutural castelhano, preferindo os sonoros helenismos do português às aspiradas arávias da língua dos tiranos." (Garrett 1826: 25$)$
} 
Literaturas nacionales, literaturas supranacionales: el lugar de los Estudios Ibéricos

inevitablemente, el ocultamiento o desvalorización de todos aquellos fenómenos o producciones que no responden a este esquema monolingüístico e ideológicamente ortodoxo, que tiene por consecuencia la separación estricta de las literaturas compuestas a uno y otro lado de la frontera ibérica.

Esta situación, sin embargo, iba a verse alterada con la aparición de nuevos impulsos de interrelación económica, política y cultural entre España y Portugal a partir de la década de 1850 y hasta aproximadamente 1930: una época de intensas relaciones hispano-lusas expresadas a través de las diversas formas de iberismo, algunas veces propiamente político, y en muchos otros casos únicamente cultural o intelectual (Sardica 2013: 24-26). Figuras influyentes de la cultura y la literatura a ambos lados de la frontera, como Oliveira Martins, Antero de Quental, Juan Valera o Menéndez y Pelayo, que no eran partidarios de la creación de un estado ibérico común, sí proponían en cambio una lectura conjunta de la historia ibérica, y en particular de su historia literaria. Es esta una época, de hecho, conflictiva y crítica para ambos estados peninsulares, en la que las identidades española y portuguesa se ven sacudidas por eventos históricos traumáticos (el Ultimatum de 1890 en el caso portugués; el Desastre de 1898 en el español) y en el que (re)surgen identidades periféricas con aspiraciones de legitimidad nacional, como la catalana, la vasca o la gallega.

Naturalmente, esta nueva sensibilidad ibérica o iberista afectó también a la historiografía literaria, si bien fue en el lado portugués donde más claramente se hizo notar. Así, ya en la Theoria da historia da litteratura portugueza de Teófilo Braga se lee: "no problema da raça nao ha hespanhoes nem portugueses. A separaçao começa na formaçao da nacionalidade” (Braga 1872: 12). Esta idea, ampliada y difundida por Antero de Quental y sobre todo por Oliveira Martins en su muy influyente Historia de la civilización ibérica (1879), mantendrá su vigencia al menos en un sector de la crítica y la historiografía portuguesa, por ejemplo en la obra de Fidelino de Figueiredo, uno de los primeros impulsores de la Literatura Comparada en Portugal:

Ha uma tradição dramatica peninsular, mas nenhuma das litteraturas a possue em toda a sua plenitude, no conjuncto do seu desenvolvimento historico. Castella, o centro geographico da Peninsula, como affirmou Pelayo, teve a gloria de possuir o momento augusto dessa tradição, mas sem a curva do desvio que para dentro da fronteira portuguesa descreve essa tradição, sem se considerar o theatro vicentino, até mesmo essa tradição [...] seria incompleta e illogica, porque o theatro de Encina, Torres Naharro e Gomes Manrique tinha caracteres estheticos diversos daquelles que, depois do impulso genial de Gil Vicente, ostentou e se encorporaram na tradição peninsular. (Figueiredo 1921: 19-20) 
PÉREZ ISASI

En España, por su parte, este iberismo civilizacional o cultural contó seguidores destacados, aunque quizás proporcionalmente no tan numerosos como en Portugal. El más conocido lusófilo del final de siglo español es, sin duda, Miguel de Unamuno (quien, sin embargo, era defensor acérrimo de la supremacía histórica castellana, en obras como En torno al casticismo), pero también un crítico tan influyente como Marcelino Menéndez y Pelayo proponía una consideración conjunta de las literaturas ibéricas en una fecha tan temprana como 1878 (un año antes de que fuera publicada la ya mencionada Historia de la civilización ibérica de Oliveira Martins):

Españoles fueron en la Edad Media los tres romances peninsulares: los tres recorrieron un ciclo literario completo, conservando unidad de espíritu y parentesco de formas en medio de las variedades locales. Eran tres dialectos hijos de la misma madre, habla dos por gentes de la misma raza, y empeñadas en común empresa. Las tres literaturas reflejaban iguales sentimientos y parecidas ideas, y recíprocamente se imitaban y traducían y cedieron el mismo paso a extrañas influencias. [...] Dios ha querido además que un misterioso sincronismo presida al desarrollo de las letras peninsulares. No hay transformación literaria en Castilla a que no responda otra igual en Lusitania. (Menéndez y Pelayo 1878)

Veamos, solo a modo de ejemplificación, otro testimonio historiográfico del último tercio del siglo XIX en que se insiste en el inextricable entrelazamiento de las literaturas española y portuguesa:

No es posible omitir en una Historia de la Literatura española los nombres de algunos poetas portugueses que escribieron en castellano, porque "siempre ha seguido Portugal el curso de la civilización española" mal que les pese a sus historiadores que regatean y escatiman la influencia que la literatura castellana ejerció en la portuguesa. (Rodríguez Miguel 1892: 298)

No quiere esto decir, naturalmente, que la tendencia historiográfica ortodoxa o centralizadora desapareciera durante el periodo de que estamos hablando: continuó vigente, en obras como la Historia de la lengua y literatura castellana de Cejador y Frauca (1915-1922). De hecho el primer tercio del siglo XX vio consolidarse ya, en la figura de Ramón Menéndez Pidal, la que sería la escuela dominante en casi todo el siglo, e incluso podría decirse que hasta nuestros días: una escuela caracterizada por la centralidad absoluta de Castilla, en lo histórico-político, en lo lingüístico y en lo literario. 
Literaturas nacionales, literaturas supranacionales: el lugar de los Estudios Ibéricos

Sin duda, la idea tan repetida de que Castilla creó a España tiene mucho de cierto, como lo tienen casi todas las ideas corrientes. No hemos de reaccionar contra ellas, desechándolas, actitud más lucida que provechosa; mejor es trabajar modestamente en reducirlas a precisión.

Es cierto que Castilla, sobre todo desde el siglo XIII, sobresalió entre las otras comarcas hermanas por ver las cosas que atañen a la vida total de España con una vehemencia y generosidad superiores, y es cierto que, desde el siglo $\mathrm{XV}$, logró y dirigió la unificación política moderna. Por eso se cree que la idea de España es una invención castellana y hasta entre los doctos en historia está arraigada la opinión de que durante la alta Edad Media no había ni asomos de un concepto unitario en la Península. (Menéndez Pidal 1929: 31)

Las páginas precedentes tienen como objetivo mostrar que existen, en la historiografía literaria española y portuguesa escrita desde comienzos del siglo XIX hasta al menos la década de 1930, diversos discursos en conflicto que reflejan diversas maneras de conceptualizar la historia, la literatura y la cultura producidas en el espacio peninsular, en función, también, de diversas configuraciones políticas e identitarias en conflicto. Este muy somero repaso permite advertir también que la historia literaria monolingüística, nacionalista o centralizadora se ve cuestionada por su incapacidad para dar cuenta de un conjunto de fenómenos que, por situarse en el límite o en los intersticios de las relaciones culturales, no encajan, ni pueden encajar, en sus esquemas explicativos. Se trata, por ejemplo, de las producciones literarias escritas en lenguas diferentes a la "lengua nacional" (los escritores hispanolatinos o hispanoárabes, por ejemplo); de los autores bilingües o que produjeron su obra en un espacio bilingüe o diglósico (como es el caso de los escritores gallegos, catalanes o vascos que alternan el español y otra lengua en sus producciones); o los autores que, sin pertenecer por origen a la nación española o portuguesa, adoptaron alguna de sus lenguas como vehículo literario (caso de los escritores portugueses que adoptaron total o parcialmente el castellano como lengua de expresión).

Es evidente, por otra parte, que estas opciones filológicas o historiográficas no son ideológicamente inocentes, sino que dependen de una determinada visión de la nación y del canon literario, así como de su función ética, social o política. En el caso español, Joan Manuel Resina (2009), entre otros, ha mostrado cómo el Hispanismo, en su desarrollo durante el Franquismo pero también en elámbito académico estadounidense, depende de una configuración nacionalista o incluso imperialista de la identidad española que desfigura, si no oculta completamente, los hechos históricos que no encajan con su particular visión del mundo. No es la menor de estas manipulaciones la extrapolación de las fronteras geopolíticas actuales a momentos del pasado (la Edad Antigua 
PÉREZ ISASI

o la Edad Media, por ejemplo) en que estas fronteras resultan anacrónicas y deturpan, más que explican, la realidad de la producción literaria. Tampoco es posible subestimar la importancia de la definición de un determinado concepto de "lo nacional" en función de un supuesto "espíritu del pueblo", una definición cargada naturalmente de ideología, que condiciona de modo radical la configuración del canon literario.

Es precisamente en el contexto de estas insuficiencias explicativas y de estos discursos en conflicto en el panorama de la historiografía ibérica, donde viene a surgir el campo de los Estudios Ibéricos por el que abogo en este texto. Los Estudios Ibéricos no son, como ya apuntaba más arriba, una mera suma de las narrativas historiográficas nacionales, sino que las superan al mismo tiempo que las incorporan; son, parafraseando la famosa cita de David Damrosch "an elliptical refraction of national literatures" (2003: 281). Naturalmente, aplicar una perspectiva ibérica a los fenómenos literarios no implica la desaparición de las ambigüedades o la inexistencia de limitaciones intrínsecas a cualquier tentativa de análisis, ni en cuanto a los límites externos del campo (¿qué es literatura ibérica, desde cuándo, desde dónde y hasta dónde existe la literatura ibérica?); pero sí elimina, al menos en nuestra configuración del nuevo campo, el peligro de una nueva re-esencialización del espacio ibérico, ya sea heredera del exotismo orientalizante del Romanticismo europeo, o de los nacionalismos hegemónicos de los siglos XIX y XX.

Los Estudios Ibéricos, de esta forma, permiten percibir la Península Ibérica como un espacio multilingüe (tanto históricamente como en la época contemporánea), con producciones, por lo tanto, en muy variadas lenguas, de las que una historia literaria que tenga espíritu abarcador, que no exhaustivo, siempre debe dar cuenta: latín, árabe, hebreo, español, catalán, gallego, vasco, etc. Iberia se presenta, también, como un espacio multicultural y plurinacional, sin que sea necesario, en este punto, entrar en discusión sobre las pretensiones de nacionalidad de Cataluña, Galicia o el País Vasco. La aproximación a tal polisistema multicultural y multilingüe plantea, desde luego, interesantes cuestiones teóricas que son también aplicables a otros ámbitos geográficos, y a espacios de otras escalas diferentes, como el europeo: $i$ Es el sistema literario ibérico uno solo, unitario, o existen sistemas literarios autónomos (hebreo, árabe y latino; español y portugués; catalán, gallego y vasco) en diversos periodos históricos o contemporáneos?

Naturalmente, el propio género de la historia literaria está una vez más en causa, porque se hace necesario (re)escribir una historia literaria que abarque y explique las diversas modulaciones de las interrelaciones entre los sistemas literarios y culturales ibéricos; que dé cuenta, también, del modo en que los textos han circulado dentro de las fronteras nacionales, pero también entre y a 
Literaturas nacionales, literaturas supranacionales: el lugar de los Estudios Ibéricos

través de estas fronteras; sin olvidar, por último, que el sistema literario ibérico se interrelaciona, también, con otros sistemas literarios supranacionales de diverso orden, y que no siempre responden, tampoco, a los esquemas explicativos filológicos tradicionales: la Romanitas, el Sur de Europa, los espacios culturales americano o africano y, por supuesto, el espacio literario y cultural europeo.

\author{
Santiago Pérez Isasi \\ santi_perez_isasi@yahoo.es \\ Centro de Estudos Comparatistas \\ Faculdade de Letras \\ Universidade de Lisboa \\ Alameda da Universidade \\ 1600-214 Lisboa \\ PORTUGAL
}

\title{
Bibliografía
}

Amador de los Ríos, J. 1861-5. Historia Crítica de la literatura española. Madrid: Imprenta de José Rodríguez.

Aullón de Haro, P. 2008. Los problemas fundamentales de la historiografía literaria moderna. - L. Tobar, ed., Literatura y nación. Zaragoza, Universidad de Zaragoza, $15-29$.

Bouterwek, F. 1829. Historia de la literatura española. Madrid: Verbum.

Braga, T. 1872. Theoria da historia da litteratura portugueza. Porto: Imprensa Portugueza.

Buffery, H., Davis, S., Hooper, K., eds. 2007. Reading Iberia: Theory/ History / Identity. Oxford: Peter Lang.

Buescu, H. 2013. Experiência do Incomum e Boa Vizinhança. Literatura Comparada e Literatura-Mundo. Porto: Porto Editora.

Damrosch, D. 2003. What is World Literature? Princeton \& Oxford: Princeton University Press.

Domínguez, C. 2006. The South European Orient: A Comparative Reflection on Space in Literary History. - Modern Language Quarterly 67 (4), 419-449.

Epps, B., Fernández Cifuentes, L., eds. 2005. Spain beyond Spain. Modernity, Literary History and National Identity. Lewisburg: Bucknell University Press.

Even-Zohar, I. 1999. Factores y dependencias en la cultura. Una revisión de la Teoría de los Polisistemas. - M. Iglesias Santos, ed., Teoría de los Polisistemas. Madrid: Arco Libros, 23-52.

Fernandes Pinheiro, J. C. 1862. Curso elementar de litteratura nacional. Río de Janeiro: Livraria de B. L. Garnier. 
PÉREZ ISASI

Figueiredo, F. 1921. Estudos de Litteratura (Terceira Série: 1918-1920). Lisboa: Clássica Editora de A. M. Teixeira.

Garrett, A. 1826. Bosquejo da história da poesia e língua portuguesa. - Obras completas de Almeida Garrett, vol. 4. Lisboa: Círculo de Leitores, 1984.

Guillén, C. 1985. Entre lo Uno y lo Diverso. Introducción a la Literatura Comparada. Barcelona: Crítica.

Lopes de Mendonça, A. P. 1849. Curso de litteratura professado no gremio litterario. Lisboa: Tipografia de Antonio José da Rocha.

Magalhães, G., ed. 2007. Actas do Congresso RELIPES III: 18, 19 e 20 de Abril de 2007. Covilhã: Universidade da Beira Interior.

Martín-Estudillo, L., Spadaccini, N., eds. 2010. New Spain, New Literatures. Nashville: Vanderbilt University Press.

Menéndez y Pelayo, M. 1878. Programa de literatura española, Estudios y discursos de crítica histórica y literaria, vol. I. - Menéndez Pelayo Digital, CD-ROM. Santander: Digibis.

Menéndez Pidal, R. 1939. La España del Cid. Buenos Aires: Espasa Calpe.

Oliveira Martins, J. P. 1879. Historia da civilização ibérica. Lisboa: Bertrand.

Pérez Isasi, S. 2013. The Limits of 'Spanishness' in Nineteenth-century Spanish Literary History. - Bulletin of Hispanic Studies 90/2, 167-188.

Pérez Isasi, S., Fernández, A. eds. 2013. Looking at Iberia. A Comparative European Perspective. Oxford: Peter Lang.

Pinheiro, T. 2013. Iberian and European Studies. Archaeology of a New Epistemological Field. - S. Pérez Isasi, A. Fernández, eds., Looking at Iberia. A Comparative European Perspective. Oxford: Peter Lang, 27-42.

Resina, J. R. 2009. Del hispanismo a los estudios ibéricos. Una propuesta federativa para el ámbito cultural. Madrid: Biblioteca Nueva.

Rodríguez Miguel, L. 1892. Compendio de historia de la literatura española. Salamanca: Imp. y Lib. de Francisco Núñez.

Sardica, J. M. 2013. Ibéria. A Relação entre Portugal e Espanha no século XX. Lisboa: Aletheia.

Schlegel, F. 1843. Historia de la literatura antigua y moderna. Barcelona: Libr. de J. Oliveres y Gavarró.

Simonde de Sismondi, J. C. L. 1813-4. De la litterature du Midi de l’Europe. París: Treuttel et Würtz.

Staël, A. L. G. 1813. De l'Allemagne. París: GF-Flammarion, 1968. 